\title{
Climate Change and Air Quality
}

Renate Forkel

Richard Knoche

Peter Suppan

Hans Peter (HaPe) Schmid

TU Munich

Institute for Meteorology \& Climate Research KIT / Research Centre Karlsruhe Garmisch-Partenkirchen \begin{abstract}
Garmisch-Partenkirchen
\end{abstract}
IMK-IFU Atmospheric Environmental Research
KIT - die Kooperation von Forschungszentrum Karlsruhe $\mathrm{GmbH}$ und Universität Karlsruhe (TH)
-

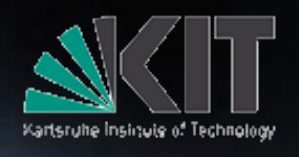
Kartsruhe Inshivis o: Technology

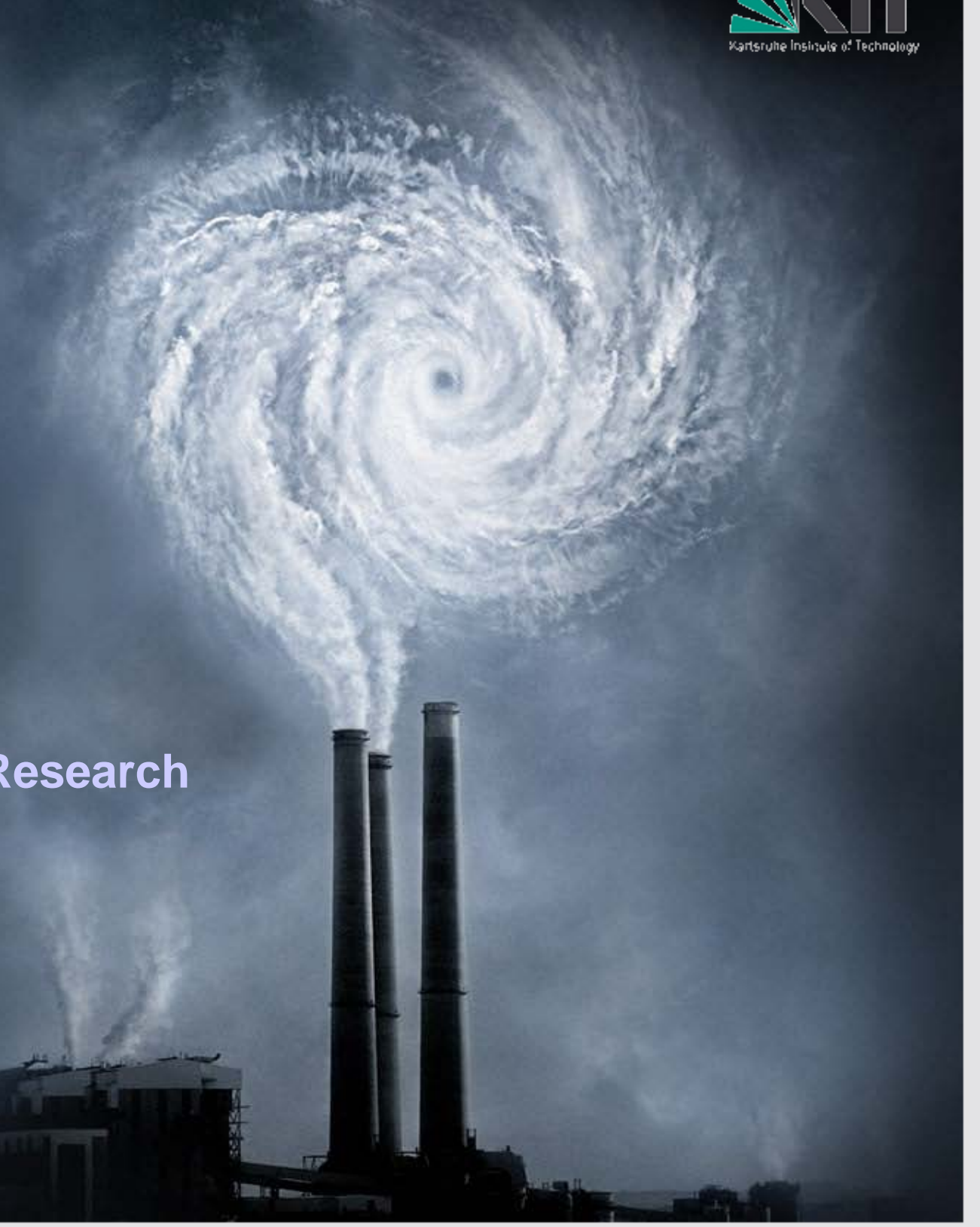

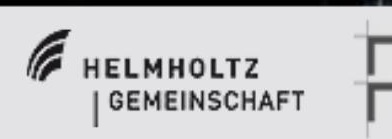

F in der Helmholtz-Gemeinschaft 


\section{Climate Change and Air Quality}

Climate Change

relates to all

Aspects of Life

\section{and Society}
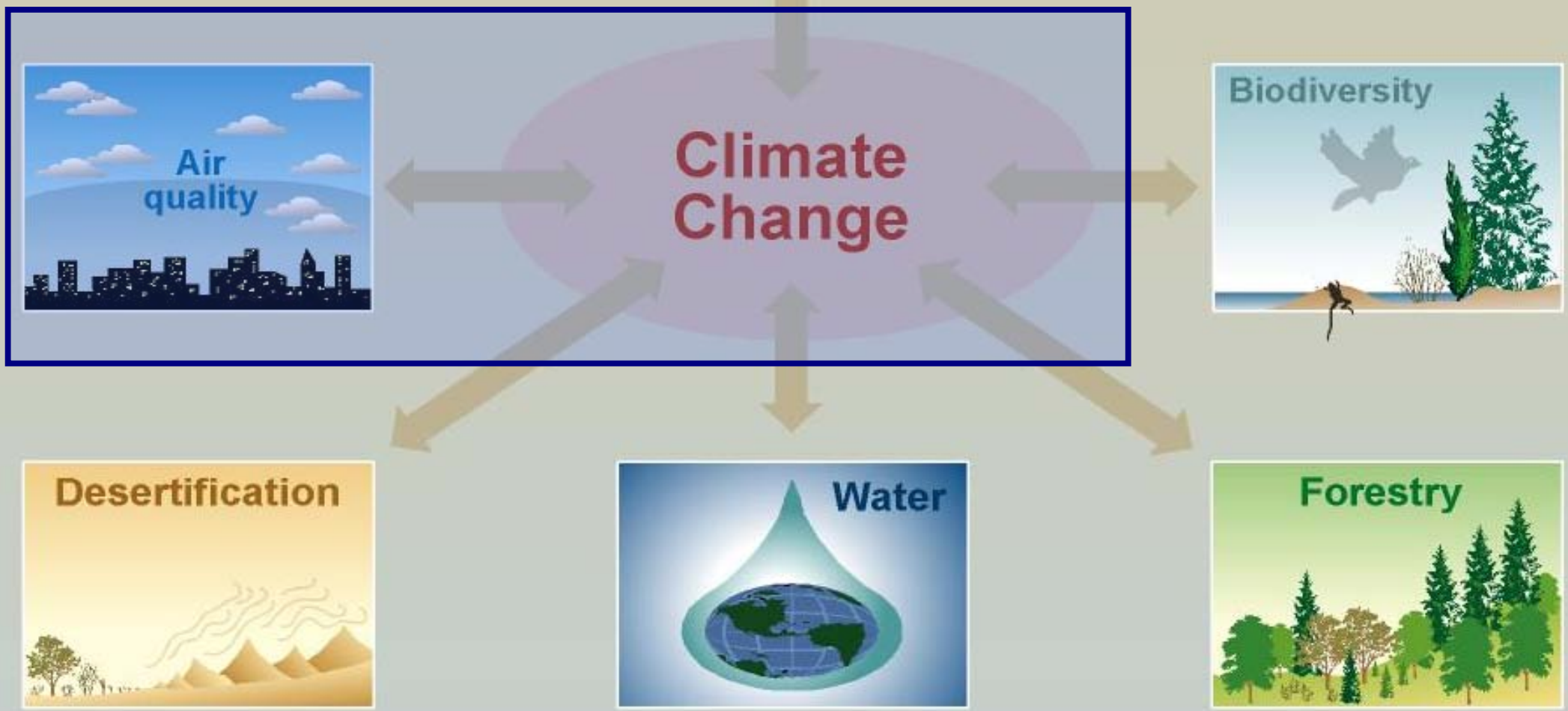

(source: IPCC 2001)

IMK-IFU Atmospheric Environmental Research Garmisch-Partenkirchen
KIT - die Kooperation von Forschungszentrum Karlsruhe $\mathrm{GmbH}$ und Universität Karlsruhe (TH) HELMHOLTZ
GEMEINSCHAFT in der Helmholtz-Gemeinschaft 


\section{Climate Change and Air Quality}

\section{Global Climate Change: Scenarios lead to Climate Projections}

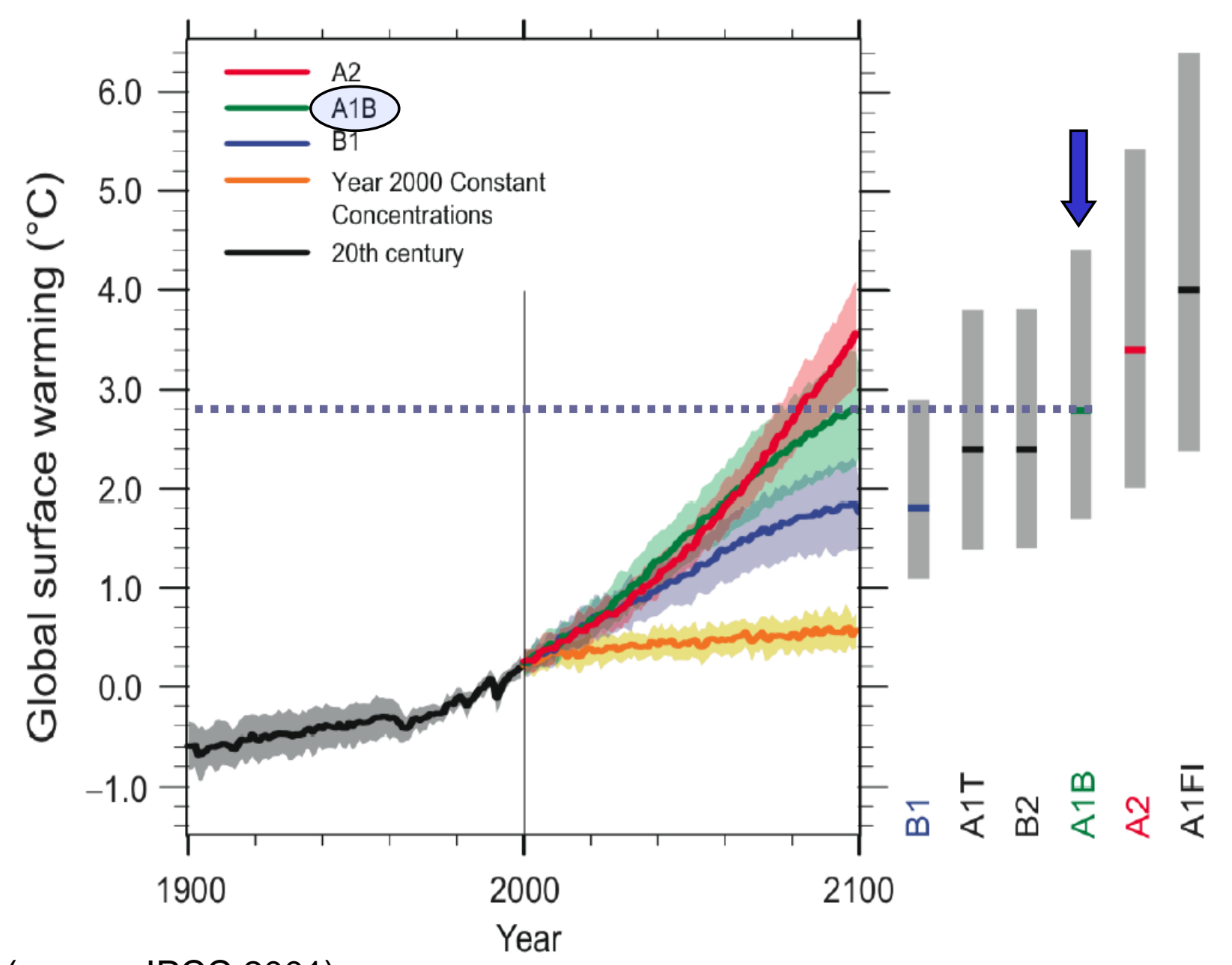

(source: IPCC 2001)

IMK-IFU Atmospheric Environmental Research Garmisch-Partenkirchen
KIT - die Kooperation von Forschungszentrum Karlsruhe $\mathrm{GmbH}$ und Universität Karlsruhe (TH)

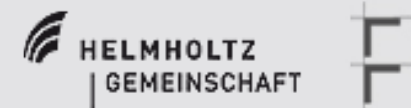




\section{Climate Change and Air Quality}

Global models indicate an increase of global mean temperature, and also for southern Germany

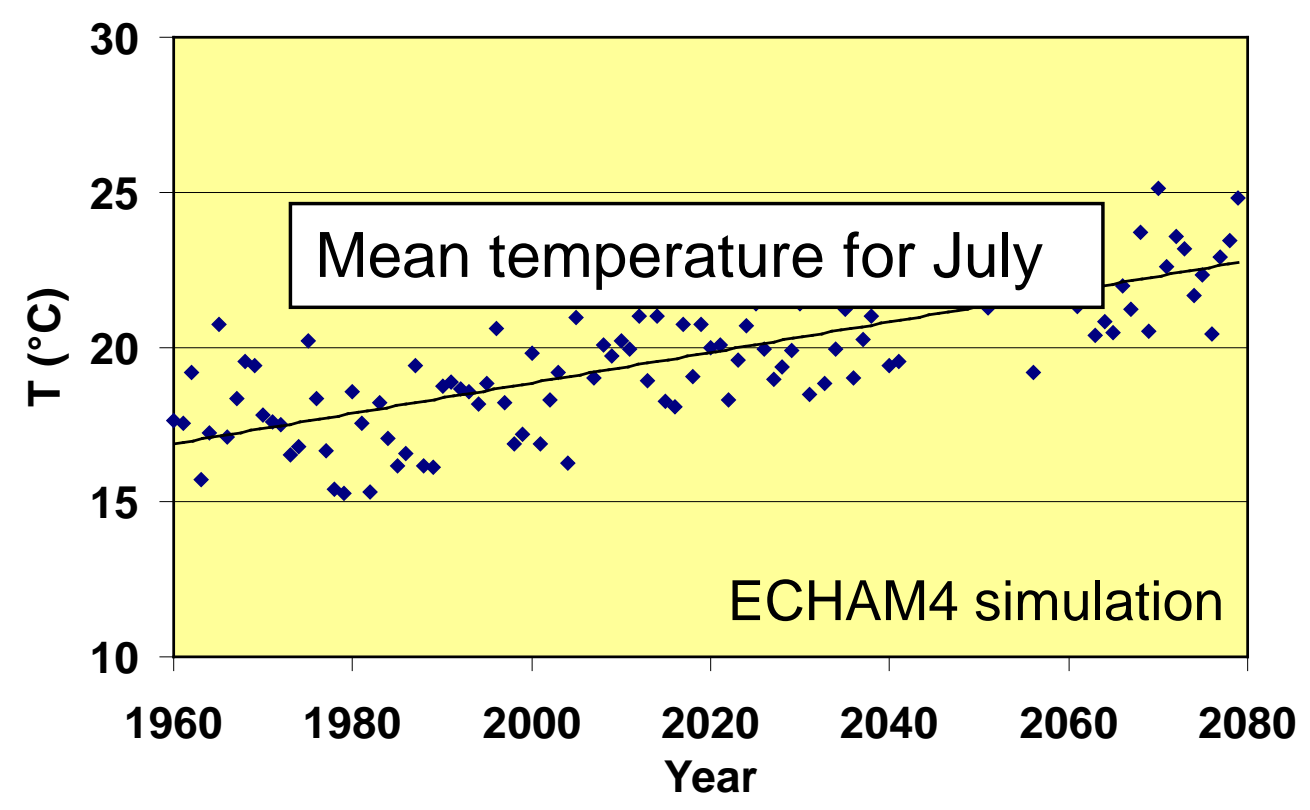

Global climate change results in regional effects on

- cloud cover, visible and UV radiation

- temperature, thermal stratification

- wind fields

- frequency and intensity of precipitation

$\longrightarrow$ Changed Climate impacts Air Quality

IMK IIIIII spheric Environmental Research Garlbayforuvtenkirchen
KIT - die Kooperation von

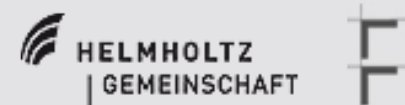




\section{Climate Change and Air Quality}

\section{Air Quality: Primarily an Urban Problem ... and Increasingly a Developing World Problem}

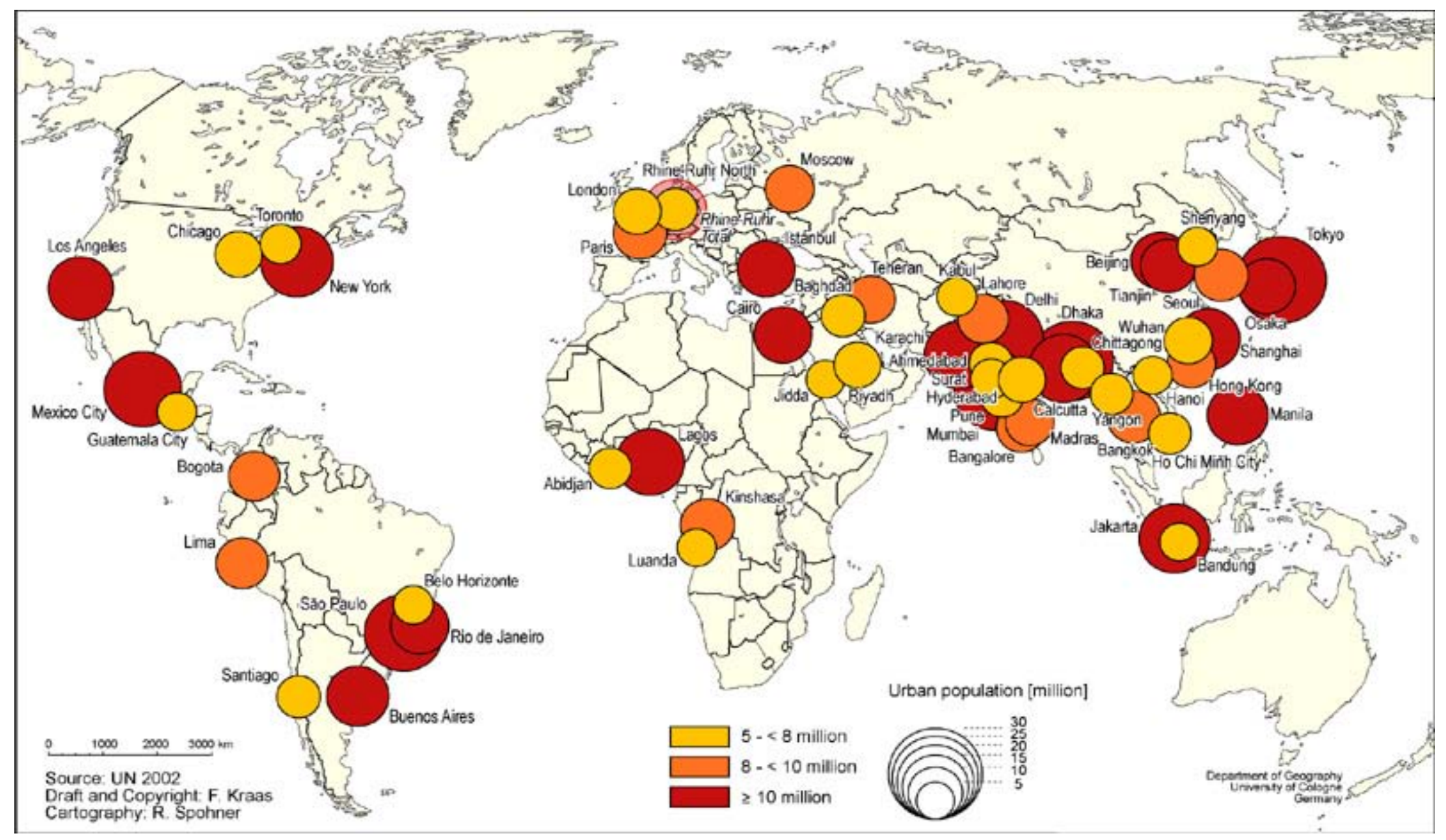

IMK-IFU Atmospheric Environmental Research Garmisch-Partenkirchen
KIT - die Kooperation von Forschungszentrum Karlsruhe $\mathrm{GmbH}$ und Universität Karlsruhe $(\mathrm{TH})$

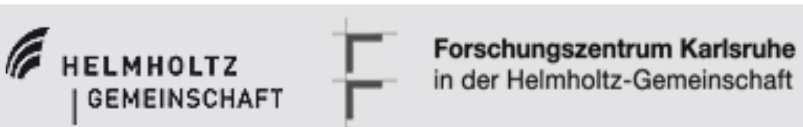




\section{Climate Change and Air Quality}

\section{Air Quality - affected by:}
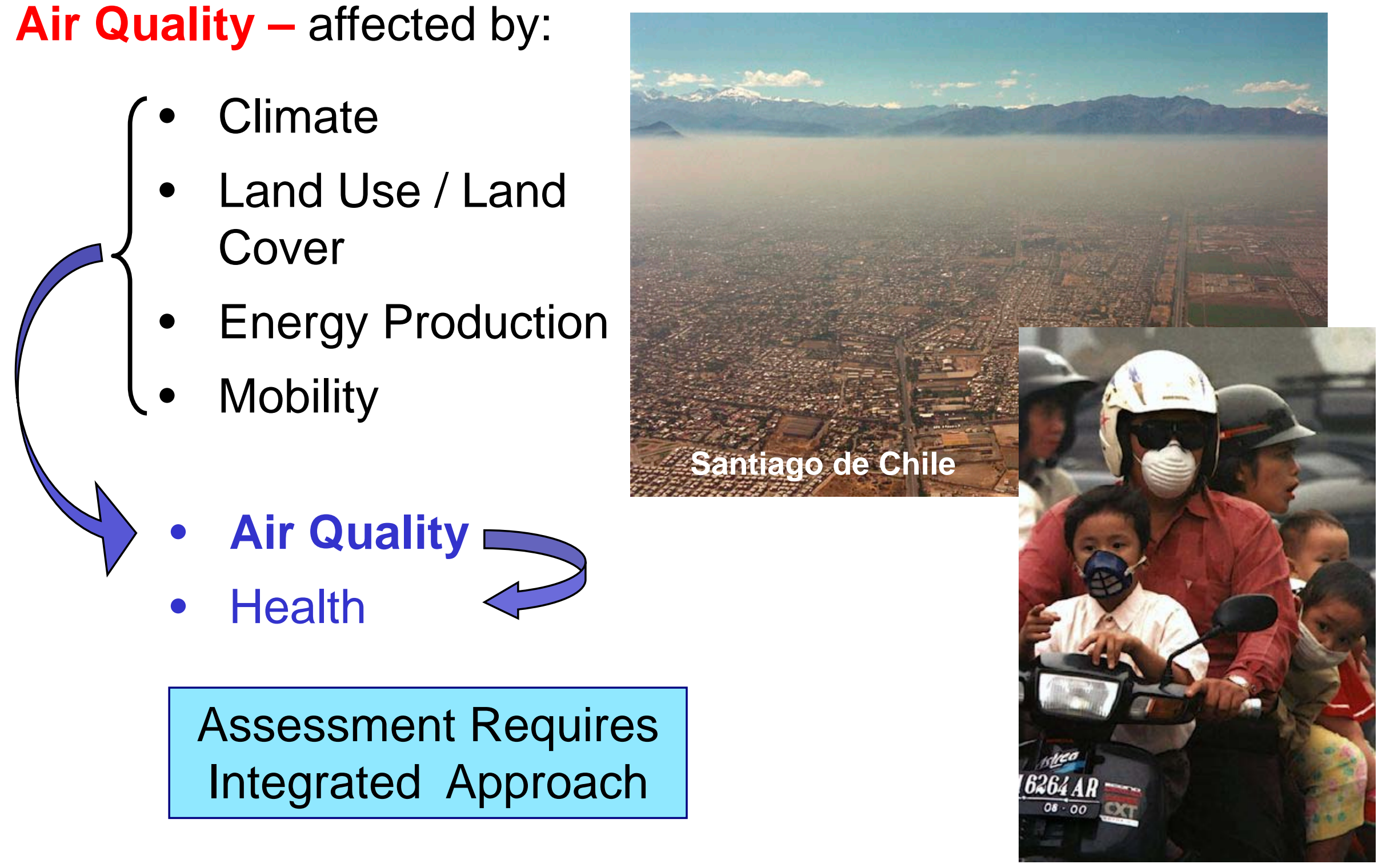

IMK-IFU Atmospheric Environmental Research Garmisch-Partenkirchen
KIT - die Kooperation von Forschungszentrum Karlsruhe $\mathrm{GmbH}$ und Universität Karlsruhe (TH)
HELMHOLTZ
GEMEINSCHAFT 


\section{Climate Change and Air Quality}

\section{Coupled Mesoscale Climate integration of models \& observations for Chemistry Model (MCCM): air quality mitigation decision support}

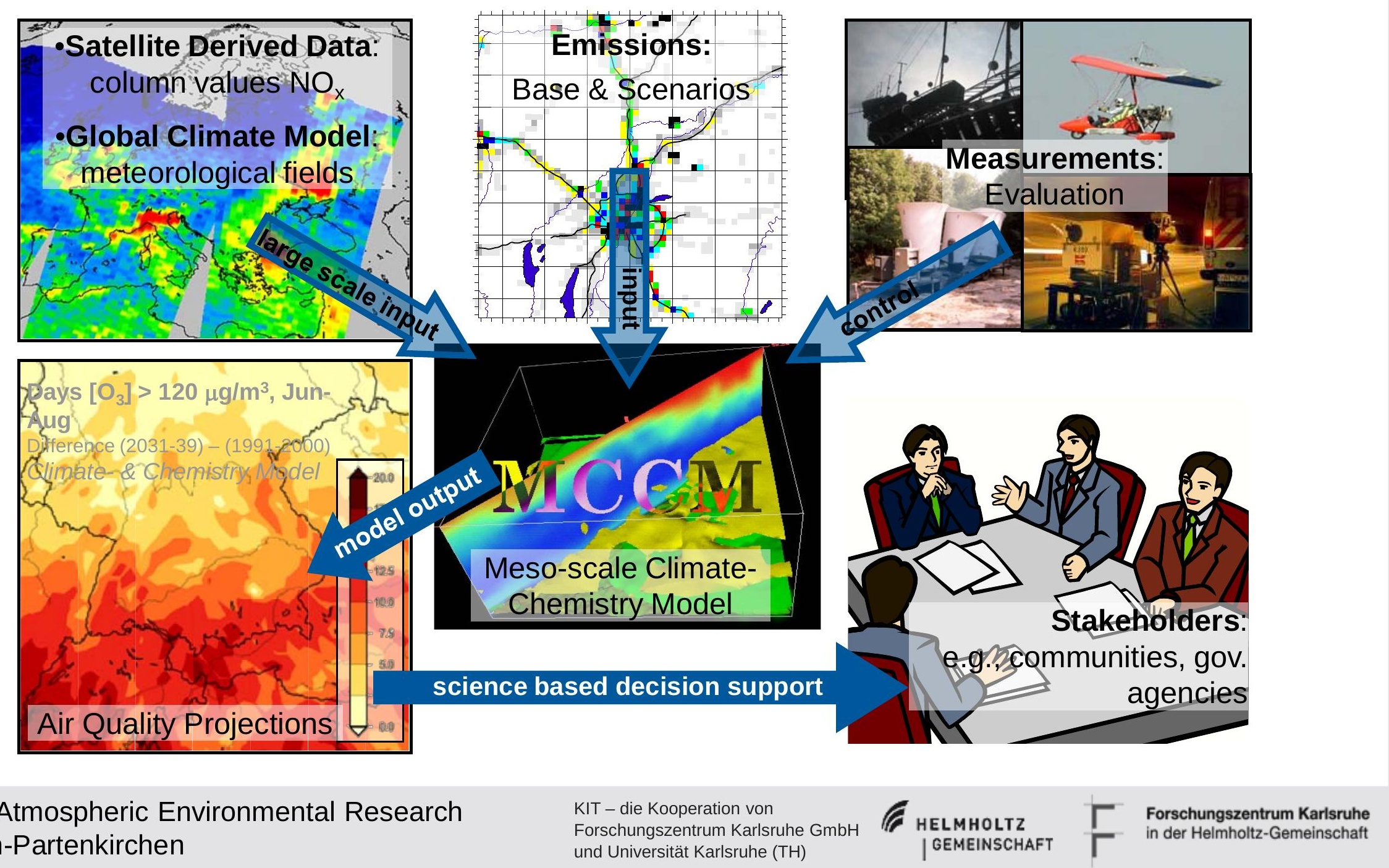

IMK-IFU Atmospheric Environmental Research Garmisch-Partenkirchen
KIT - die Kooperation von und Universität Karlsruhe ( $\mathrm{TH}$ ) 


\section{Climate Change and Air Quality Effect of land use changes}

\section{Application to Mexico City}

Mexico City

Population

Urbanized area $\left(\mathrm{km}^{2}\right)$

Population density $\left(\mathrm{p} / \mathrm{km}^{2}\right)$

Population growth (\%/ y)

\section{Estimated expansion by 2010}

$\rightarrow$ stronger Urban Heat Island

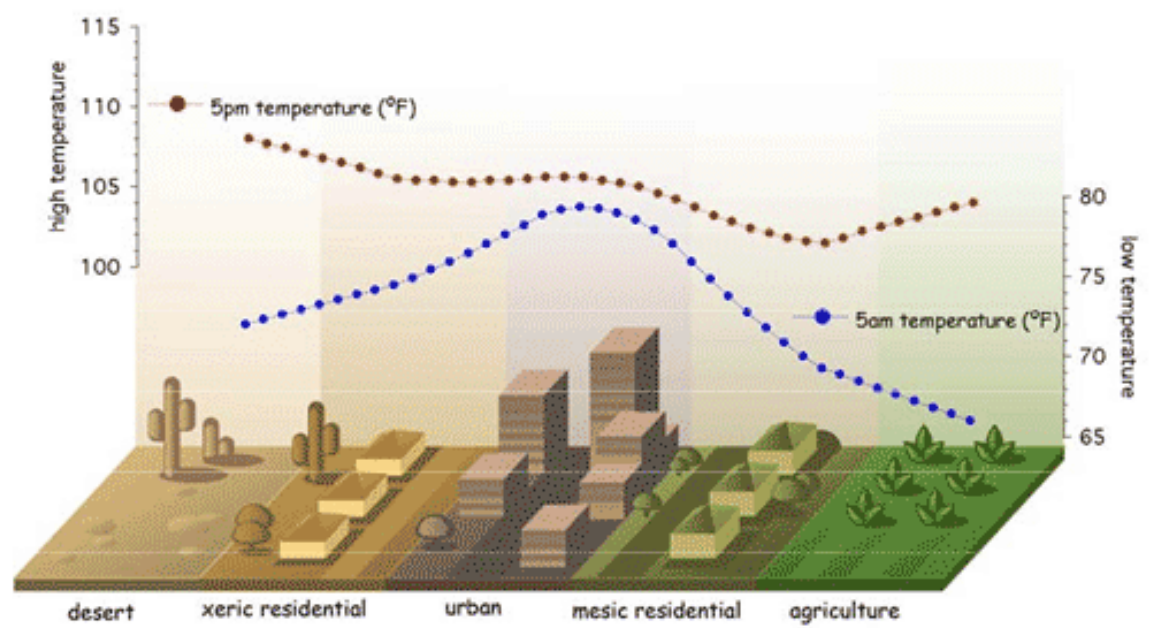

(http://censam.mit.edu)

IMK-IFU Atmospheric Environmental Research Garmisch-Partenkirchen
$19,410,000$ 1800

10,800

$\sim 1,28$

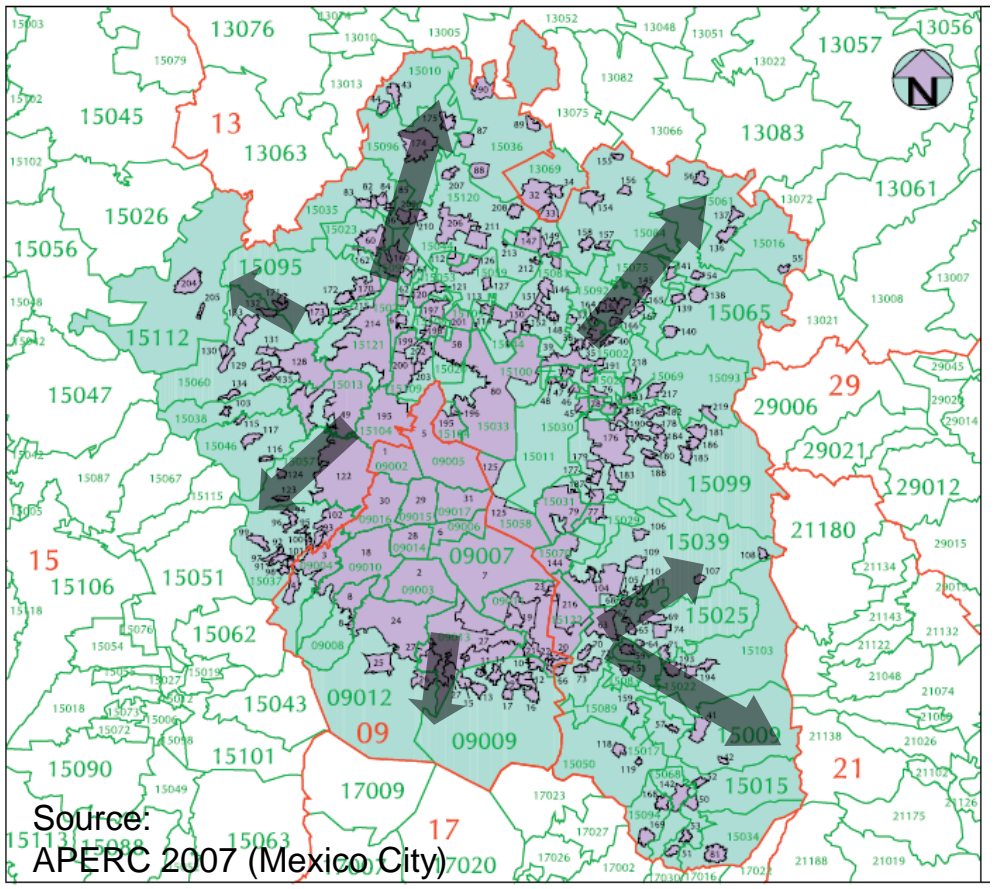

Land Use Change leads to Regional Climate Change 


\section{Climate Change and Air Quality Effect of land use changes}

\section{Application to Mexico City}

Temperature difference with and without urban sprawl

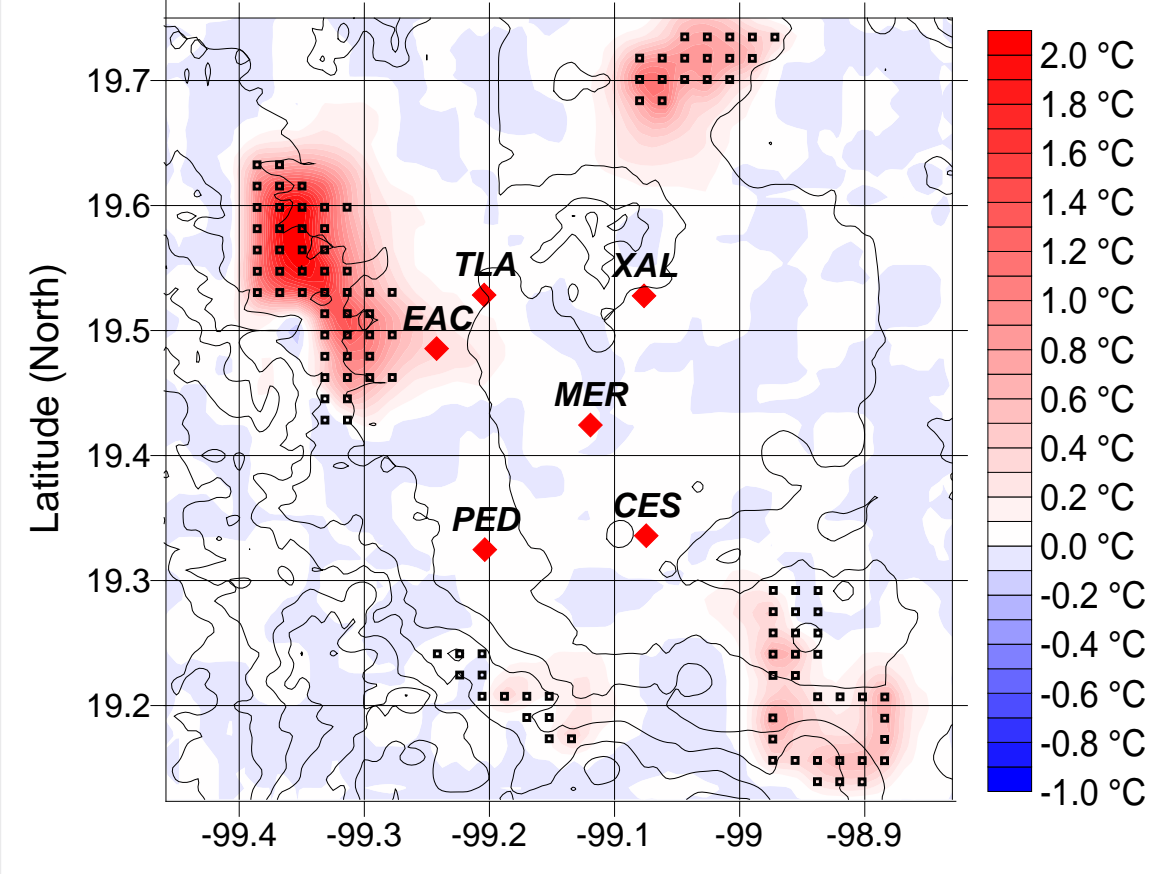

(source: Forkel) Longitude (East)

land use change combines with climate change: can enhance or offset air quality ramifications

IMK-IFU Atmospheric Environmental Research Garmisch-Partenkirchen
KIT - die Kooperation von Forschungszentrum Karlsruhe $\mathrm{GmbH}$ und Universität Karlsruhe (TH)

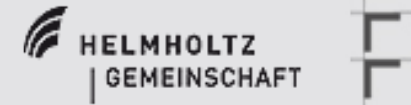




\section{Climate Change and Air Quality}

\section{Regional Climate Change in Southern Germany}

Temperature

Temperature $\left({ }^{\circ} \mathrm{C}\right)$ Jun-Aug

Difference 2031/2039 - 1991/2000 uv20

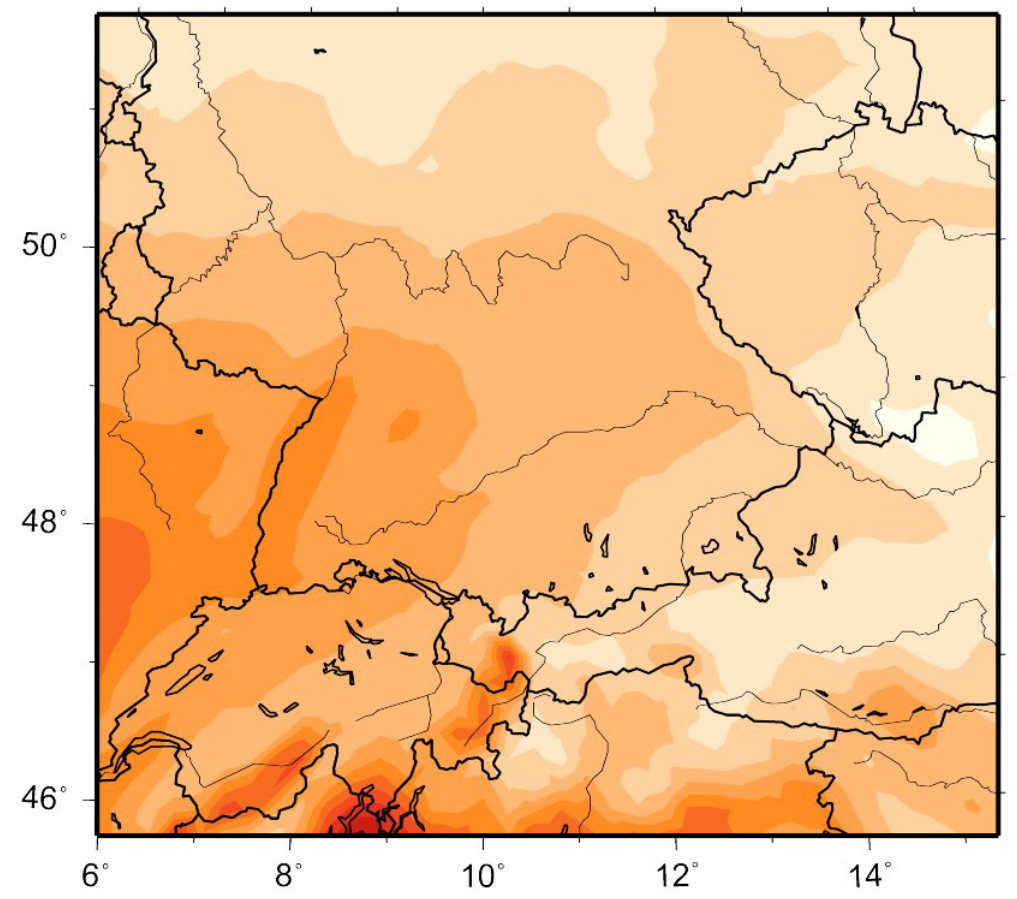

(Forkel \& Knoche, 2006. JGR)

\section{Cloud Water}

Cloud Water Content $\left(\mathrm{g} / \mathrm{m}^{2}\right)$ Jun-Aug Difference 2031/2039 - 1991/2000 uv20

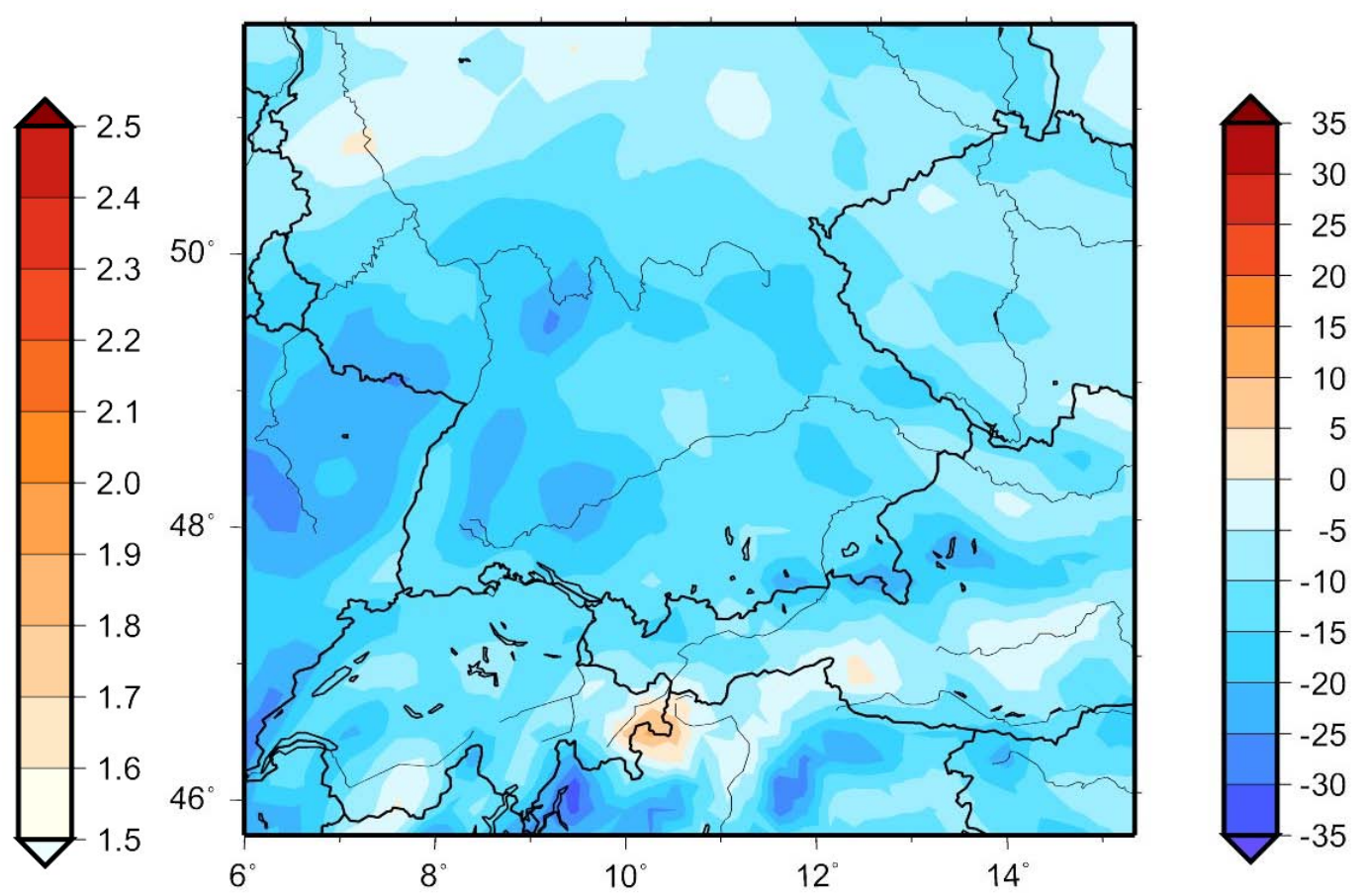

IMK-IFU Atmospheric Environmental Research Garmisch-Partenkirchen
KIT - die Kooperation von Forschungszentrum Karlsruhe $\mathrm{GmbH}$ und Universität Karlsruhe (TH)
HELMHOLTZ
GEMEINSCHAFT $\quad \begin{aligned} & \text { Forschungszentrum Karlsruhe } \\ & \text { in der Helmholtz-Gemeinschaft }\end{aligned}$ 


\section{Climate Change and Air Quality}

\section{Regional Climate Change in Southern Germany}

\section{Solar Radiation}

Solar Radiation $\left(\mathrm{W} / \mathrm{m}^{2}\right)$ Jun-Aug Difference 2031/2039 - 1991/2000 uv20

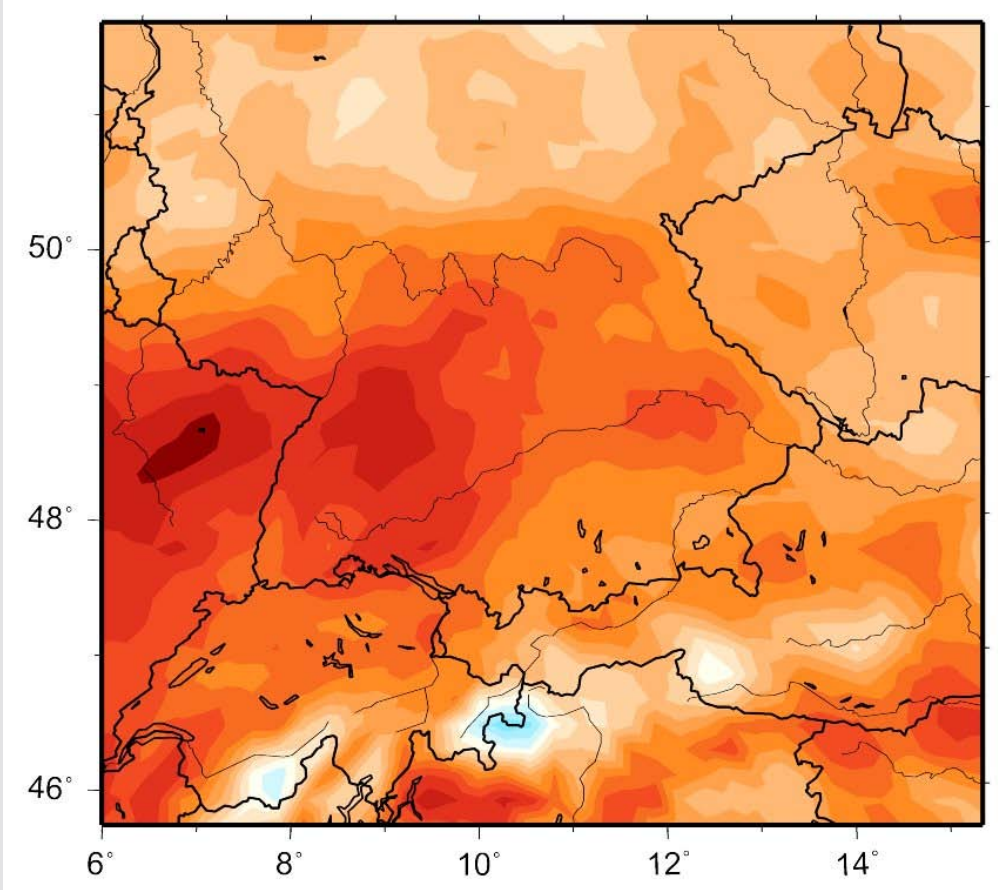

(Forkel \& Knoche, 2006. JGR)

\section{UV Radiation}

UV-Radiation $\left(\mathrm{mW} / \mathrm{m}^{2}\right)$ Jun-Aug Difference 2031/2039 - 1991/2000 uv20

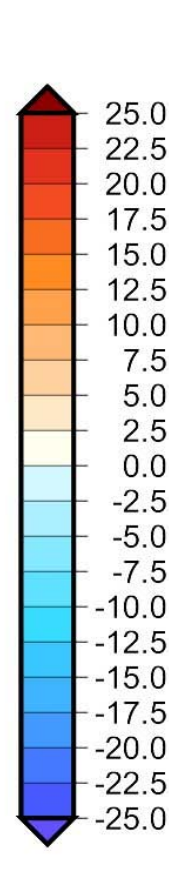

IMK-IFU Atmospheric Environmental Research Garmisch-Partenkirchen
KIT - die Kooperation von Forschungszentrum Karlsruhe $\mathrm{GmbH}$ und Universität Karlsruhe (TH)
HELMHOLTZ
GEMEINSCHAFT i $\begin{aligned} & \text { Forschungszentrum Karlsruhe } \\ & \text { in der Helmholtz-Gemeinschaft }\end{aligned}$ 


\section{Climate Change and Air Quality}

\section{Regional Air Quality change in Southern Germany}

\section{Isoprene Emission} Isoprene emission $\left(\mu \mathrm{g} / \mathrm{m}^{2} / \mathrm{min}\right)$ Jun-Aug Difference 2031/2039-1991/2000 uv20

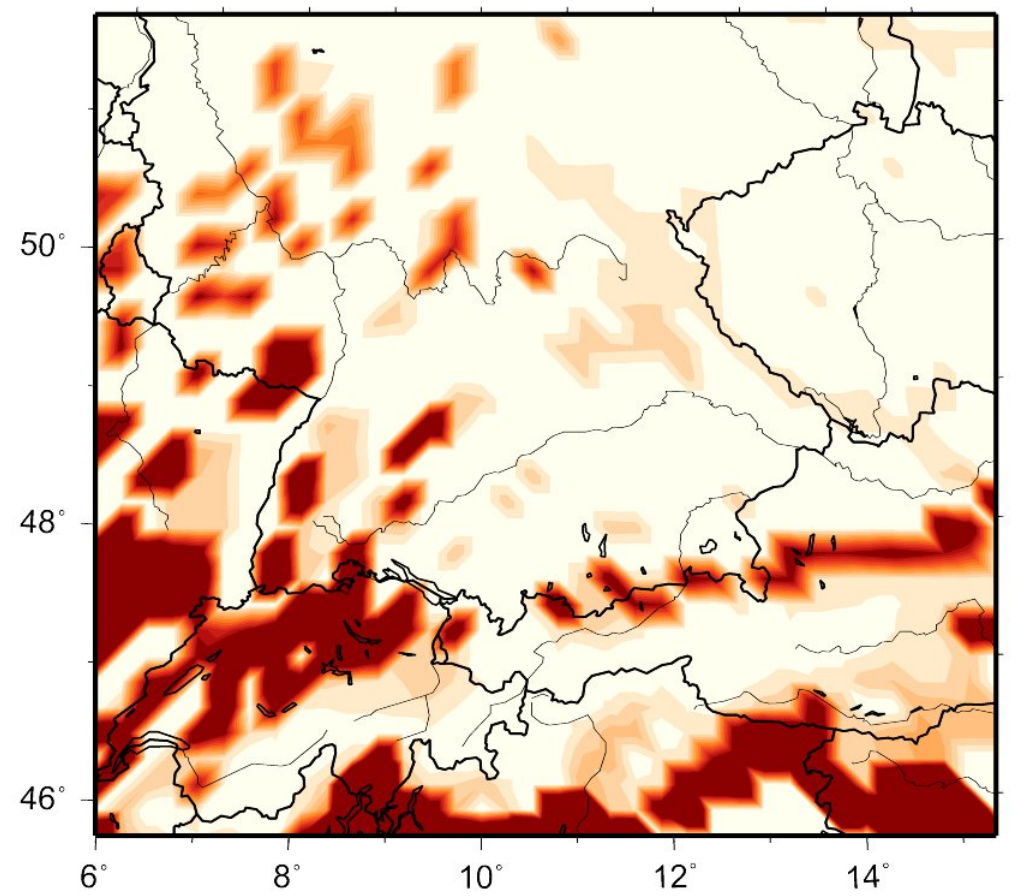

(Forkel \& Knoche, 2006. JGR)

\section{Soil NO Emission}

NO emission $\left(\mu \mathrm{g} / \mathrm{m}^{2} / \mathrm{min}\right)$ Jun-Aug

Difference 2031/2039 - 1991/2000 uv20

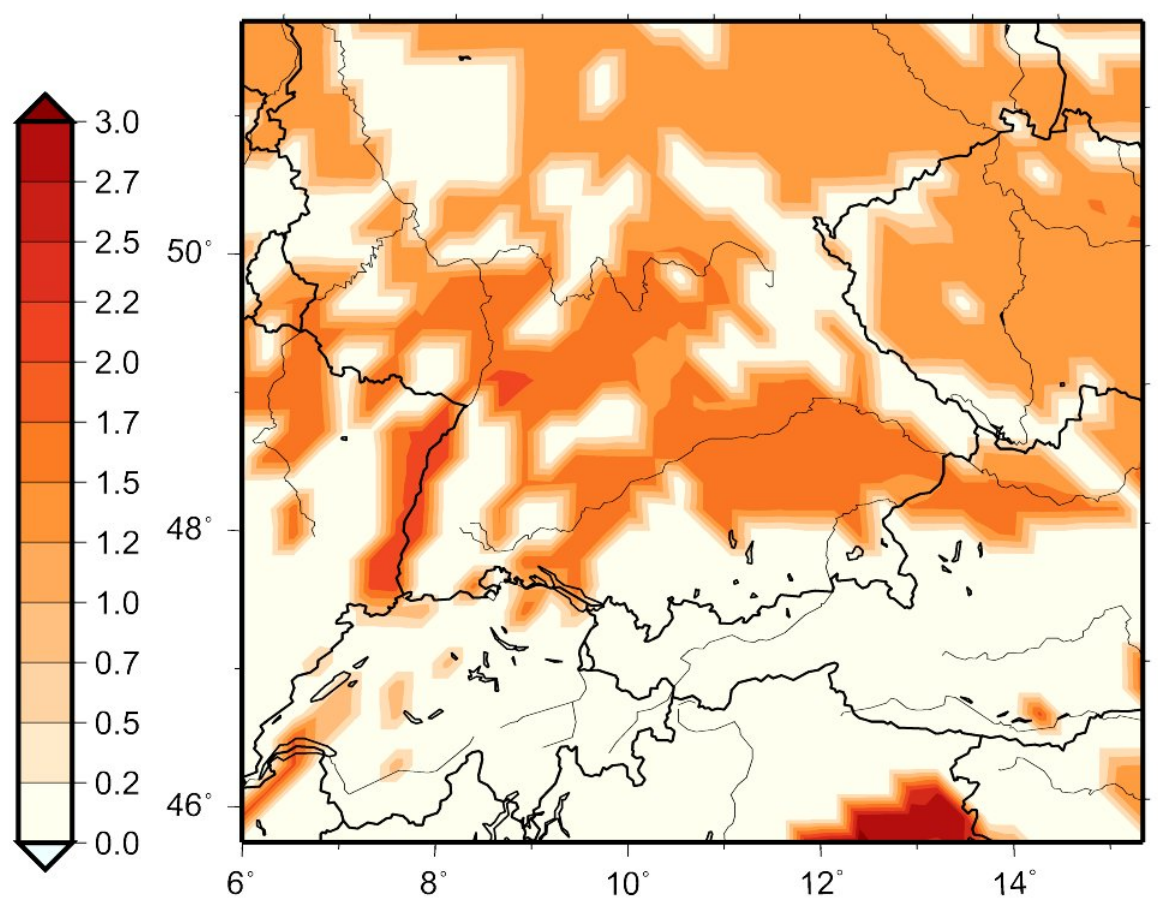

IMK-IFU Atmospheric Environmental Research Garmisch-Partenkirchen
KIT - die Kooperation von Forschungszentrum Karlsruhe $\mathrm{GmbH}$ und Universität Karlsruhe (TH)
HELMHOLTZ
GEMEINSCHAFT $\quad \begin{aligned} & \text { Forschungszentrum Karlsruhe } \\ & \text { in der Helmholtz-Gemeinschaft }\end{aligned}$ 


\section{Climate Change and Air Quality}

\section{Regional Air Quality change in Southern Germany}

\section{Isoprene Mixing Ratio}

Isoprene (ppb) Jun-Aug

Difference 2031/2039 - 1991/2000 uv20

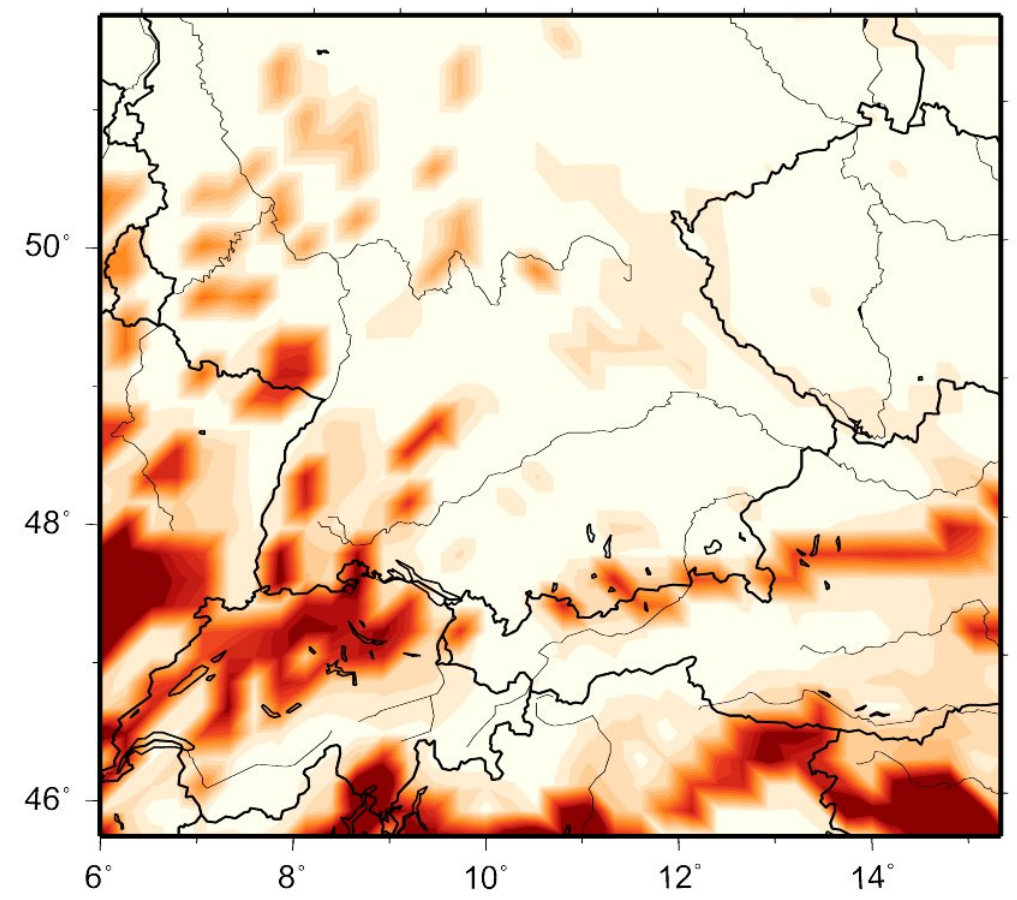

(Forkel \& Knoche, 2006. JGR)

IMK-IFU Atmospheric Environmental Research Garmisch-Partenkirchen

\section{$\mathrm{NO}_{\mathrm{x}}$ Mixing Ratio}

$\mathrm{NO}_{\mathrm{x}}(\mathrm{ppb})$ Jun-Aug

Difference 2031/2039 - 1991/2000 uv20

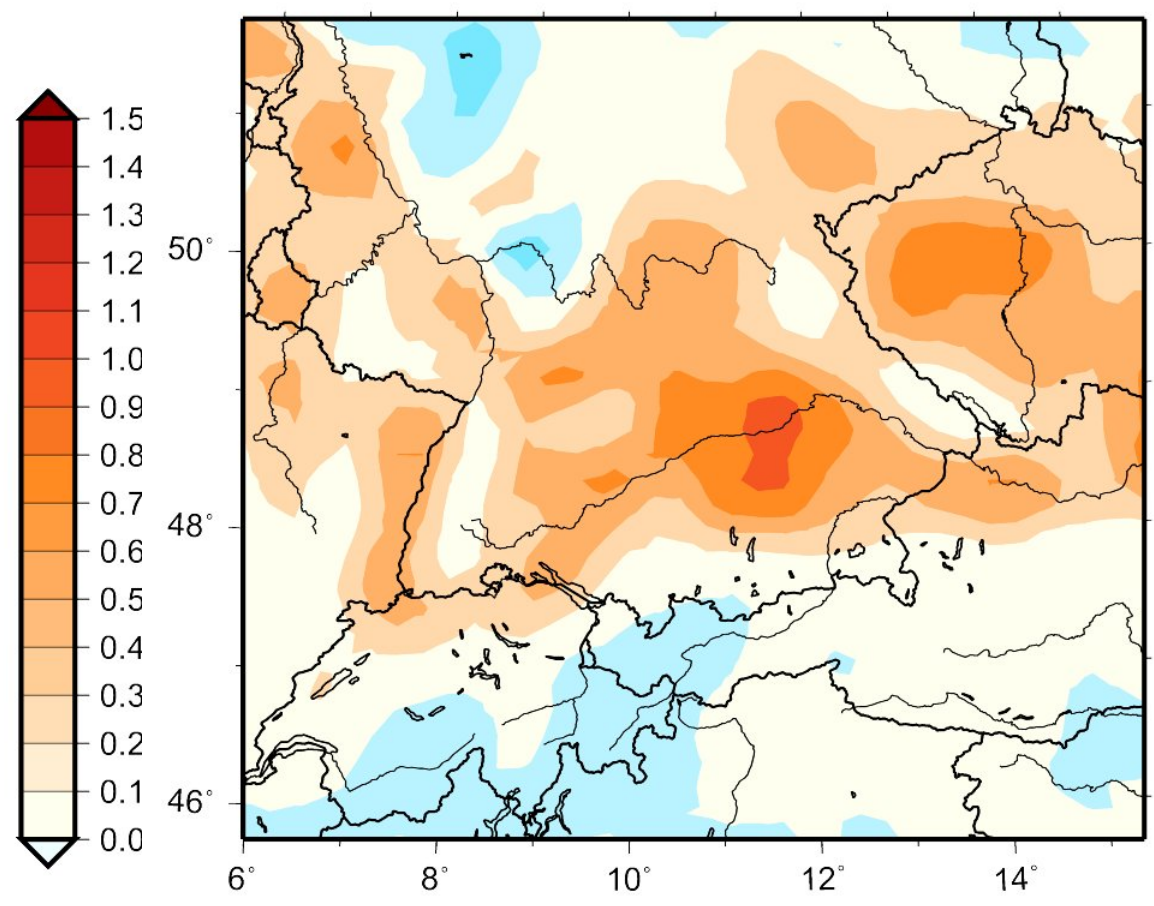

3.0
2.5
2.0
1.5
1.0
0.5
0.0
-0.5
-1.0
-1.5
-2.0
-2.5
-3.0
KIT - die Kooperation von Forschungszentrum Karlsruhe $\mathrm{GmbH}$ und Universität Karlsruhe (TH)
HELMHOLTZ
GEMEINSCHAFT 


\section{Climate Change and Air Quality}

\section{Regional Air Quality change in Southern Germany}

\section{Ozone Maximum}

Daily Ozone Maximum (ppb) Jun-Aug

Difference 2031/2039 - 1991/2000 uv20

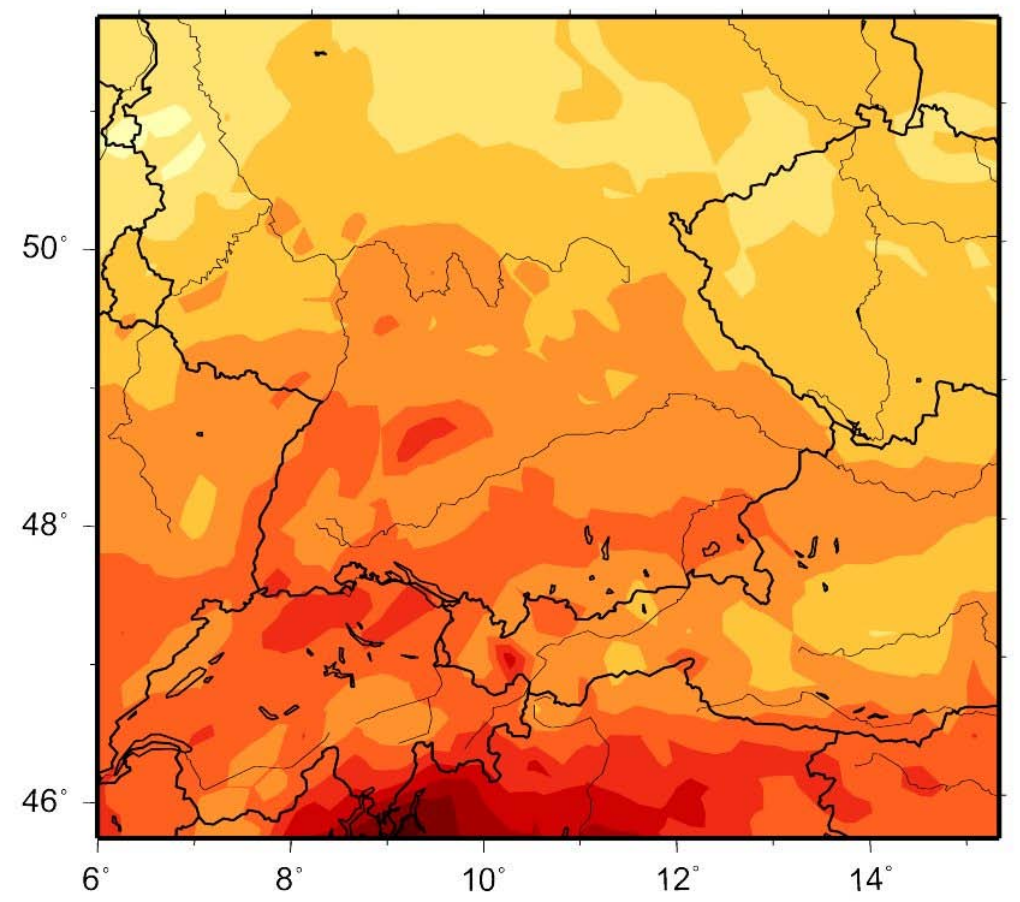

(Forkel \& Knoche, 2006. JGR)

IMK-IFU Atmospheric Environmental Research Garmisch-Partenkirchen
$\mathrm{O}_{3}$ Exceedance Days

Days with Threshold Exceedance Jun-Aug Difference 2031/2039 - 1991/2000 uv20

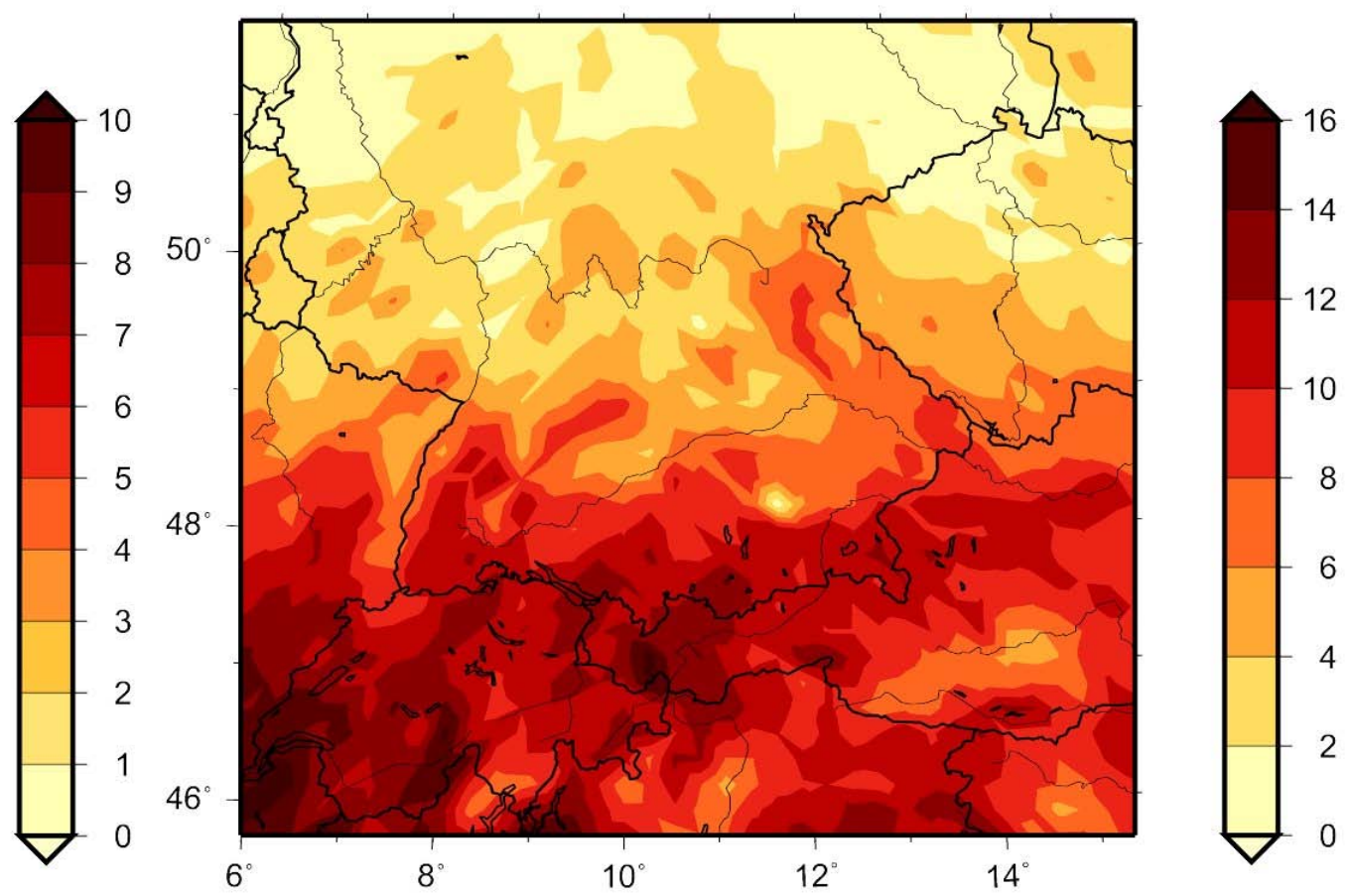

KIT - die Kooperation von Forschungszentrum Karlsruhe $\mathrm{GmbH}$ und Universität Karlsruhe (TH)
HELMHOLTZ
GEMEINSCHAFT i $\begin{aligned} & \text { Forschungszentrum Karlsruhe } \\ & \text { in der Helmholtz-Gemeinschaft }\end{aligned}$ 


\section{Climate Change and Air Quality}

\section{Regional Air Quality change in Southern Germany Distribution of daily Ozone Maxima}
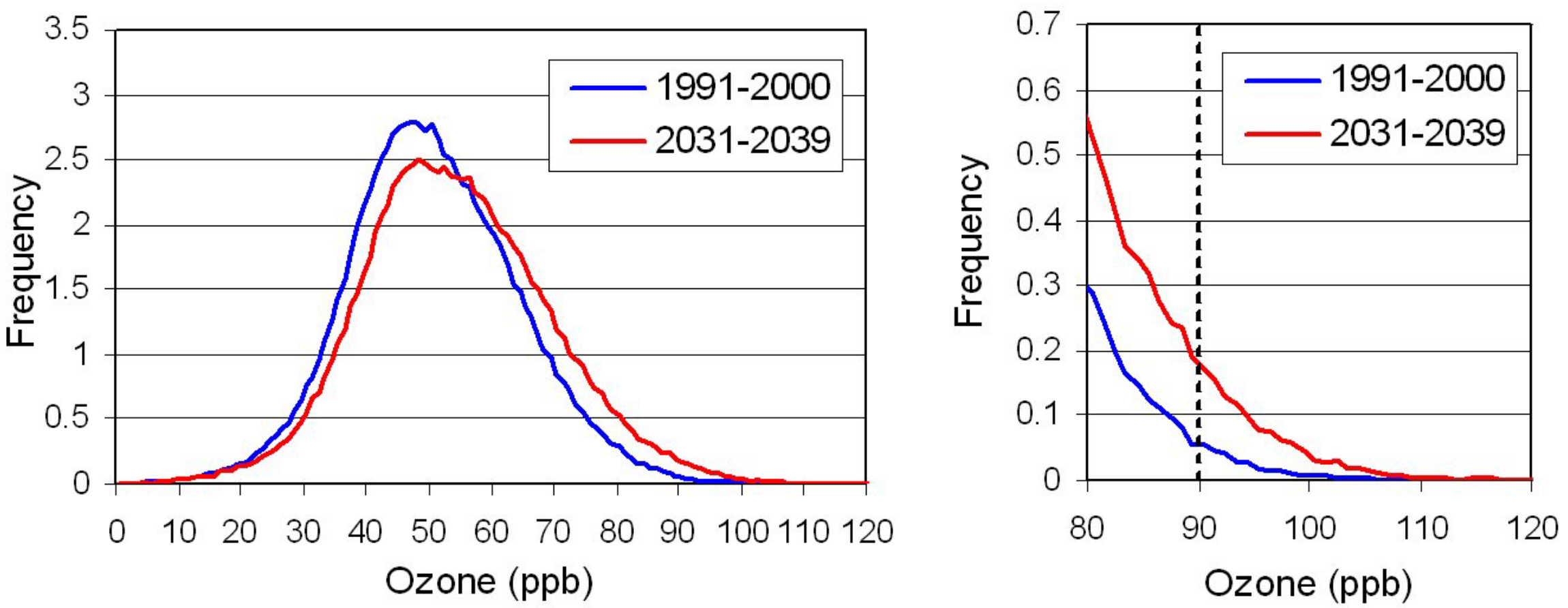

(Forkel \& Knoche, 2006. JGR)

\section{Occurrence of $\max .\left[\mathrm{O}_{3}\right]>180 \mu \mathrm{g} / \mathrm{m}^{3}$ increases 4-fold}

IMK

spheric Environmental Research

Garlbayforuvtenkirchen
KIT - die Kooperation von Forschungszentrum Karlsruhe $\mathrm{GmbH}$ und Universität Karlsruhe (TH) $\underset{\substack{\text { helmholtz } \\ \text { GeMEINSCHAFt }}}{\text { T }}$

Forschungszentrum Karlsruhe in der Helmholtz-Gemeinschaft 


\section{Climate Change and Air Quality}

\section{Summary of Results}

- global climate change has pronounced regional expression (e.g., Alps, Urban Areas)

- land use change can amplify or offset climate change

- climate change affects thermal / humidity / radiation environment in which air pollutants evolve (and precursors are emitted)

- climate change can strongly affect air quality at a regional scale $\left(-10^{2}-10^{3} \mathrm{~km}\right)$

- Example - Northern Pre-Alpine (D, CH, A): expect 4-fold Number of Ozone Days

IMK-IFU Atmospheric Environmental Research Garmisch-Partenkirchen
KIT - die Kooperation von Forschungszentrum Karlsruhe $\mathrm{GmbH}$ und Universität Karlsruhe (TH)
HELMHOLTZ
|GEMEINSCHAFT 


\section{Climate Change and Air Quality}

\section{Thank you for your attention!}

This work is supported by the German Federal Ministry of Education and Research (BMBF) through the Helmholtz Association.

IMK-IFU Atmospheric Environmental Research Garmisch-Partenkirchen
KIT - die Kooperation von

Forschungszentrum Karlsruhe $\mathrm{GmbH}$ und Universität Karlsruhe (TH)

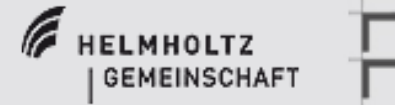

Forschungszentrum Karlsruhe in der Helmholtz-Gemeinschaft 